\title{
Synchronous primary ovarian and endometrial cancers: a series of cases and a review of literature
}

\author{
Sylwia Dębska-Szmich ${ }^{1}$, Urszula Czernek ${ }^{1}$, Magdalena Krakowska ${ }^{1}$, Marta Frąckowiak², Agnieszka Zięba², \\ Rafał Czyżykowski ${ }^{1}$, Dominika Kulejewska², Piotr Potemski ${ }^{1}$
}

${ }^{1}$ Klinika Chemioterapii Nowotworów, Katedra Onkologii, Uniwersytet Medyczny w Łodzi

${ }^{2}$ Klinika Chemioterapii Nowotworów, Wojewódzki Szpital Specjalistyczny im. Kopernika w Łodzi

\begin{abstract}
Synchronous cancers account for $0.7-1.8 \%$ of all gynecologic cancers. Among them, synchronous ovarian and endometrial cancers are predominant (40-53\%). Patients with synchronous cancers have better prognosis than those with single disseminated cancer. We present 10 patients with synchronous ovarian and endometrial cancers who were treated at the Chemotherapy Department of the Medical University of Lodz in 2009-2013. The most often reported symptom of the disease was abnormal vaginal bleeding ( 6 patients). The range of the patients' age was 48-62 and the median age was 56 . Five patients had stage I of ovarian cancer, single patients had stage IIA, IIB and IIIB, 2 patients had stage IIIC. Three patients had I, 5 - II, and 2 - III stage of endometrial cancer. All patients had endometrioid type of endometrial cancer, 7 of them had also the same histological type of ovarian cancer. All patients had adjuvant chemotherapy because of ovarian cancer, none of them had adjuvant radiotherapy. One patient was lost to follow up. For other patients a median follow up was 13 months (range: 3-53 months). One patient experienced relapse, all patients are alive. Synchronous ovarian and endometrial cancers are usually diagnosed at an earlier stage, have lower histological grade and better prognosis than single cancers. The most common histological type of both endometrial and ovarian cancers is endometrioid carcinoma. The first symptoms reported by our patients and the course of the disease were concordant with data from the literature.
\end{abstract}

Key words: synchronous cancers, ovarian cancer, endometrial cancer, endometrioid type, adjuvant treatment.

\section{Introduction}

Synchronous neoplasms occur within one year, if there is a longer time between their occurrence, they are metachronic and earlier oncologic treatment is considered as a probable carcinogen. Synchronous primary malignancies account for $0.7-1.8 \%$ of all gynecologic tumors [1-3]. Most common synchronous ovarian and endometrial cancers $[3,4]$ comprise $40-53 \%$ of all synchronous gynecologic malignancies. Synchronous ovarian and endometrial cancers are found in $3.3-5 \%$ of all patients with endometrial cancer and in $2.7-10 \%$ of all patients with ovarian cancer $[5,6]$ and even in $12-50 \%$ of patients with ovarian cancer of endometrioid histology.

Because endometrioid histology is the most common in both localizations, differentiation between 3 clinical situations is often necessary: primary endometrial cancer with metastases to ovaries, primary ovarian cancer with metastases to endometrium or two synchronous primary cancers. A precise diagnosis is important because these 3 situations are related to different prognosis [5] and require different postoperative management. We present 10 patients with synchronous ovarian and endometrial cancers.

\section{Cose 1}

In October 2008, a 59-year-old woman experienced postmenopausal vaginal bleeding and mild pelvic pain. She presented with a firm, hardly movable mass of $15 \mathrm{~cm}$ in diameter localized in the lower abdomen. CT scan revealed a capsulated, heterogeneous tumor localized in the left part of pelvis. Serum concentration of CA125 was $48.86 \mathrm{U} / \mathrm{ml}$. Typical panhysterectomy, omentectomy and appendectomy were carried out. After the surgery, serum concentration of CA125 normalized. Histopathological examination revealed endometrioid cancer G1 of the left ovary (stage IC) and endometrioid cancer G1 of uterus in stage IIIB. According to the pathologist, equivocal identification of the origin of the cancer was not possible. A diagnosis of synchro- 
nous primary ovarian and endometrial cancers was established.

The patient was subsequently treated with adjuvant chemotherapy - she received 6 cycles of carboplatin and paclitaxel. The last observation was recorded in March 2013. She was free of recurrence 51 months after surgery.

\section{Case 2}

In August 2012, a 48-year-old woman was admitted to the emergency room because of acute severe pain in the abdomen lasting for several hours. Physical examination revealed a firm, hardly movable tumor of $20 \mathrm{~cm}$ in diameter originating from the pelvis. The abdomen was painful on palpation especially in its right lower part; peritoneal signs were absent.

Ultrasound (US) examination showed a partially solid and partially cystic pathologic mass of $20 \times 12 \mathrm{~cm}$ in size, up to the left epigastrium.

An emergency surgery was performed. The patient was intraoperatively diagnosed with bilateral ovarian tumors and a tumor of uterus, moreover a rupture of the tumor of the right ovary was found. Typical panhysterectomy, omentectomy and appendectomy were performed - the surgery was macroscopically radical.

Histopathological examination revealed bilateral endometrioid adenocarcinoma G2 in ovaries (stage IC) and endometrioid adenocarcinoma G3 in uterus in stage pT2NO (II).

One month after the operation, serum concentration of CA125 was normal. The patient was treated with 6 cycles of adjuvant chemotherapy with carboplatin and paclitaxel. After completion of the treatment she was lost to follow up.

\section{Case 3}

In December 2011, a 57-year-old woman experienced postmenopausal vaginal bleeding lasting for several weeks. In 2006, she had been treated with chemotherapy because of high grade large B-cell lymphoma of a tonsil.

She presented with a firm, hardly movable mass of $15 \mathrm{~cm}$ in diameter localized in the lower abdomen. US examination revealed anteflexed uterus which size was $83 \times 63 \mathrm{~mm}$, thickness of endometrium was $34 \mathrm{~mm}$. There was a partially solid and partially cystic tumor of $45 \mathrm{~mm}$ in diameter at the site of the right ovary. Serum concentration of CA125 was $884 \mathrm{U} / \mathrm{ml}$.

In January 2012, she was operated. Histopathological examination revealed bilateral serous cystic adenocarcinoma $\mathrm{G} 3$ in ovaries in stage IIA and endometrioid adenocarcinoma $\mathrm{G} 2$ in uterus in stage IA.

The patient was subsequently treated with 6 cycles of adjuvant chemotherapy with carboplatin and paclitaxel. She was free of recurrence 14 months after surgery.

\section{Case 4}

In May 2012, a 52-year-old woman was admitted to the gynecologic department because of synchronous tumors of the right ovary and the uterus in order to undergo a planned surgery. Physical examination revealed a firm, hardly movable, polycyclic tumor of $25 \mathrm{~cm}$ in diameter originating from the pelvis. US examination showed a partially solid and partially cystic pathologic mass in the pelvis, its size was $13 \times 10 \mathrm{~cm}$.

Standard panhysterectomy, partial omentectomy and appendectomy were carried out.

Histopathological examination confirmed endometrioid adenocarcinoma G2 of the right ovary (stage IA) and endometrioid adenocarcinoma $G 2$ of uterus (stage IA).

Clinical and pathological data indicated that the patient suffered rather from two synchronous primary malignancies than endometrial cancer with metastasis to ovary.

Three months after the surgery, serum concentration of CA125 was normal. She received 5 cycles of carboplatin and paclitaxel - the chemotherapy was discontinued because of severe peripheral polyneuropathy. She was free of recurrence 12 months after surgery.

\section{Case 5}

In August 2011, a 62-year-old woman was admitted to the gynecologic department because of ascites, significant loss of body weight $(20 \mathrm{~kg})$ and nausea. US examination revealed ascites, the pelvis was filled with a huge heterogeneous tumor. Serum concentration of CA125 was $748.1 \mathrm{U} / \mathrm{ml}$.

Panhysterectomy, omentectomy and appendectomy were performed.

Histopathological examination confirmed bilateral mucinous adenocarcinoma $G 2$ of the ovaries involving parametrium (stage IIB) and endometrioid adenocarcinoma $\mathrm{G} 2$ of uterus in stage II.

The patient was subsequently treated with 6 cycles of adjuvant chemotherapy with carboplatin and paclitaxel.

She was free of recurrence 16 months after surgery.

\section{Case 6}

In March 2013, a 55-year-old woman was admitted to the gynecologic department because of a large ovarian tumor. She complained of the pain of her left leg and a rapid loss of the body weight $-10 \mathrm{~kg}$ during 1 month. In a physical examination she presented with a huge tumor filling the whole abdomen. Serum concentration of CA125 was $678.3 \mathrm{U} / \mathrm{ml}$.

Panhysterectomy, paraaortic lymphadenectomy, appendectomy and omentectomy were performed.

Histopathological examination revealed undifferentiated carcinoma G3 originating from rhabdomyoid 
cell with fields of neuroendocrine differentiation of the left ovary. Its immunohistochemical profile was: CKAE1/AE3 - weak positivity in isolated cells, chromogranin - focally positive, synaptophysin - focally positive, LCA $-(-)$, desmin - (-), CD $138-(+/-)$, PAX $5-(-)$, CD $56-(-)$, calretinin $-(-)$, CD30 - (-), CK7 - (-), CK $20-(-)$, PAX 8 - (-). In uterus, endometrioid adenocarcinoma $G 2$ was found. There were also metastases of undifferentiated carcinoma (similar to the ovarian cancer) of $1 \mathrm{~cm}$ in maximal diameter in the uterus and in one paraaortic node. The patient was diagnosed with synchronous ovarian cancer IIIC and endometrial cancer II. X-ray of the chest detected leftsided hydrothorax up to the 5. rib and the widened left upper mediastinum. Serum concentration of CA125 was $124.1 \mathrm{U} / \mathrm{ml}$. She started chemotherapy with carboplatin and paclitaxel, but after the fourth cycle she complained of relapse of the severe pain in the left leg. CT scan revealed a huge pathologic mass around the lumbar spine infiltrating vertebrae on the left side. Currently she is being diagnosed to identify the histopathologic type of this pathologic mass.

\section{Case 7}

In October 2012, a postmenopausal 55-year-old woman experienced a vaginal bleeding. Histopathological evaluation of the endometrium specimen confirmed endometrioid adenocarcinoma.

Subsequently panhysterectomy, appendectomy, omentectomy and pelvic lymphadenectomy were carried out.

Histopathological examination confirmed endometrioid adenocarcinoma G2 with focal planoepithelial metaplasia of uterus and bilateral endometrioid adenocarcinoma G2 with diffuse planoepithelial metaplasia of the ovaries. Due to similar appearance of the cancers, presence of planoepithelial neoplasia in both localizations, lack of endometriosis and lack of WT1 expression in both tumors, the pathologist suggested that it could be one metastatic cancer. But the early stage of the cancer in uterus indicated that it should be two synchronous neoplasms. Finally, she was diagnosed with synchronous ovarian cancer (stage IC) and endometrial cancer (stage II).

The patient was treated with 6 cycles of adjuvant chemotherapy with carboplatin and paclitaxel. CT scan performed after completion of the treatment revealed a hypodense lesion of $8 \times 10 \mathrm{~mm}$ in size located in the fourth segment of the liver. Currently the character of the lesion is being verified.

\section{Case 8}

In November 2011, a 57-year-old woman was admitted to the gynecologic department because of a sus- picion of an ovarian cancer. CT scan displayed a cystic tumor $(11 \times 7.2 \mathrm{~cm})$ of the left ovary.

Panhysterectomy, appendectomy and omentectomy were performed. The surgery was macroscopically radical.

Histopathological examination revealed endometrioid adenocarcinoma G2 of the left ovary (stage IA) and endometrioid adenocarcinoma G2 with planoepithelial differentiation of the uterus (stage IA). Regarding early stages of both tumors, the pathologist diagnosed two primary synchronous neoplasms.

Postoperative serum concentration of CA125 was normal.

Six cycles of adjuvant chemotherapy with carboplatin and paclitaxel were planned, but after the fourth cycle she suffered from severe anemia. Because of the side effects and patient's reluctance to continue the treatment, chemotherapy was discontinued. She was free of recurrence 17 months after the operation.

\section{Case 9}

In January 2012, a 53-year-old woman experienced an abnormal vaginal bleeding and a mild pelvic pain. She presented with a firm, hardly movable, painful on palpation tumor of $20 \mathrm{~cm}$ in diameter localized in the lower abdomen.

Panhysterectomy, omentectomy, pelvic and paraaortic lymphadenectomy were carried out.

Histopathological examination revealed bilateral endometrioid adenocarcinoma G1 of ovaries and endometrioid adenocarcinoma G1 of the uterus.

The patient was diagnosed with synchronous ovarian cancer IIIC and endometrial cancer IIIC2.

Postoperative serum level of CA125 was $59.3 \mathrm{U} / \mathrm{ml}$. She was treated with 6 cycles of adjuvant chemotherapy with carboplatin and paclitaxel. After the treatment serum concentration of CA125 normalized. The patient was free of recurrence 15 month after surgery.

\section{Case 10}

In April 2013, a 59-year-old woman experienced a pain in the lower abdomen and intermittent constipations.

US examination and CT scan displayed a partially cystic and partially solid tumor of $10 \mathrm{~cm}$ in diameter located in rectouterine pouch. Serum concentration of CA125 was $98.23 \mathrm{U} / \mathrm{ml}$.

Panhysterectomy, omentectomy, and appendectomy were carried out.

Histopathological examination revealed endometrioid cancer G1-2 of the left ovary staged IIIB and endometrioid cancer G1 of the uterus staged II. Regarding similar morphology of both tumors it was difficult to equivocally diagnose two synchronous primary cancers and exclude metastatic endometrial cancer. 
Postoperative serum concentration of CA125 was normal.

Six cycles of chemotherapy with carboplatin and paclitaxel were scheduled - currently she is on treatment.

\section{Discussion}

A distinction between synchronous primary ovarian and endometrial cancers and one disseminated cancer is based on the following pathological criteria described by Scully et al. [6] and employed by pathologists at our institution:

1) histological dissimilarity of tumors (in 3 our patients);

2) no or only superficial myometrial invasion of endometrial tumor;

3) no vascular space invasion of endometrial tumor;

4) atypical endometrial hyperplasia additionally present;

5) absence of other evidence of spread of endometrial tumor;

6) ovarian tumor unilateral (80-90\% of cases, but in our series only $50 \%$ );

7) ovarian tumors located mainly in parenchyma;

8) no vascular space invasion, surface implants, or predominant hilar location in the ovary;

9) absence of other evidence of spread of ovarian tumor;

10) ovary endometriosis present.

Clinical course of the disease is also considered [7].

Currently the value of some molecular features like loss of heterozygosity $(\mathrm{LOH})$, clonal inactivation of chromosome $\mathrm{X}$ or microsatellite instability $(\mathrm{MI})$ in the diagnostic process is being explored but still there are no unequivocal data. For example, different patterns of $\mathrm{LOH}$ in two tumors not necessarily indicate their different clonal origin, as they may represent distant fragments of the same heterogeneous tumor. On the other hand, identical patterns of chromosome $\mathrm{X}$ inactivation were found in two different clones of cells in $50 \%$ of examined cases [7].

Mutations of PTEN/MMAC1 (ch10q23) are often seen in patients with type I endometrial cancer [8]. Endometrioid adenocarcinoma is the most common histological type in patients with synchronous primary ovarian and endometrial cancers [9, 10]. Lin et al. [8] suggest that the same mutations of PTEN and $\mathrm{LOH}$ in $10 q 23$ region in both ovarian and endometrial tumors indicate that they are one disseminated cancer. For example hormonal factors may play a role in tumorigenesis [12]. Therefore, pathological features remain the basis of the diagnostic process and the value of molecular and genetic markers is still unknown [7].

Synchronous primary ovarian and endometrial cancers are diagnosed at a younger age and they are seen 2-10 times more frequently than it could be expected regarding the morbidity rate of single ovarian or endometrial cancer. These data suggest some genetic predispositions for synchronous cancers [5].
Both ovarian and endometrial cancers are typical of Lynch syndrome - a disease that results from a defect in DNA mismatch repair genes (MMR), which leads to the microsatellite instability [13]. Soliman et al. [13] screened 102 patients with synchronous ovarian and endometrial cancers for typical mutations of $\mathrm{MSH} 2, \mathrm{MSH}$, MLH1. $7 \%$ of them met molecular or clinical criteria of Lynch syndrome - they had a history of previous cancer typical of the syndrome or affected first-degree relative. This study showed that synchronous ovarian and endometrial cancers in most cases are related to other genetic or environmental factors than Lynch syndrome. For example, mutations of the TP53 gene or polymorphism of the MDM2 gene, which encodes down regulator of $p 53$, are engaged in pathogenesis of endometrial cancer [10, 14]. Thus, molecular testing should be limited to women with a family or a personal history of previous cancer distinctive for the syndrome. In our study, none of the patients had documented Lynch syndrome.

According to the literature, most patients with synchronous cancers present symptoms characteristic of endometrial cancer [15]. The most frequently reported symptoms are abnormal vaginal bleeding (42-70\%), pain in lower abdomen (17-44\%), palpable tumor in pelvis (28$40 \%$ ) and increased serum concentration of CA125 (65\%) $[6,9,16-20]$.

In our series, abnormal vaginal bleeding was observed in 6 patients, palpable pelvic tumor - in 5 patients, abdominal pain - in 4 patients, painful leg - 1 patient, loss of body weight -2 patients and constipation - 1 patient. An increased serum concentration of CA125 was observed in 6 cases.

As mentioned before, synchronous cancers occur in younger patients than single cancers [5]. Oranratanaphan et al. [21] compared clinical and pathological features as well as survival in patients with the synchronous cancers and single endometrial cancer disseminated to ovaries. Patients with synchronous cancers were younger (aged 47 vs. 56). According to other authors, a median age of patients with the synchronous tumors was 47-55 [6, 16-20].

In our series, the range of the patients' age was 48-62 and the median age was 56 . For comparison, in the population of patients with ovarian cancer from the district of Lodz, the median age was 52 (range 25-79) [22]

In Oranratanaphan's et al. [21] study, patients with the synchronous cancers were diagnosed at earlier stages comparing with patients with a single cancer. They also had lower histological grading. All patients with synchronous cancers had stage I of endometrial cancer and $85 \%$ of them had also early stage of ovarian cancer.

According to other authors, stages of both malignancies were for ovarian cancer: IA - 11-71\%, IB - 9\%, IC - 16-39\%, II - 9-22\%, III - 21\%, and for uterine cancer: IA - 37-42\%, IB - 32-47\%, IC - 4\%, II - 8\%, IIIA $-9 \%$, IIIC $-9 \%[6,16-20]$. In $56 \%$ of the patients, both neoplasms were diagnosed at stage I. 
In our series of patients, the stage was:

- for ovarian cancer: IA in 2 patients, IC in 3 patients, IIA, IIB and IIIB in single patients, IIIC - in 2 patients;

- for uterine cancer: IA in 3 patients, II in 5 patients, IIIB and IIIC in single patients.

In 2 patients, both cancers were diagnosed at IA stage. In our district, the patients with single ovarian cancer were diagnosed at stages: I - 15\%, II - 9\%, III $64 \%$, IV - 12\% [22].

Endometrioid cancer is a rare histological type of ovarian cancer and it is thought to develop under the same conditions as endometrial cancer. According to Signorelli et al. [18], in patients with the synchronous cancers endometrioid type or mixed type with an endometrioid component is predominant. Ma et al. reported that this histological type was found in $69.8 \%$ of patients in the ovary. $46-88 \%$ of the patients had endometrioid carcinoma both in the uterus and in the ovary $[16,17,19,20]$.

Endometriosis of ovaries may play a role in the development of endometrioid ovarian cancer. According to some authors, endometriosis of ovaries coexisted with endometrioid ovarian cancer in $22-59 \%$ of patients [ 6 , $18,20]$.

In our series, endometrioid histology in the ovary was observed in 7 of 10 patients. Other types of ovarian cancer were: papillary cystadenocarcinoma, mucinous adenocarcinoma and undifferentiated carcinoma. For comparison, in the patients with single ovarian cancer from our district, the endometrioid type was diagnosed only in $14.3 \%$ of cases and serous histology prevailed (30.7\%) [21]. All our patients with synchronous cancers had endometrioid cancer in endometrium.

Surgery is mandatory in operable stages of endometrial and ovarian cancers. Optimal extent of the operation in endometrial cancer covers panhysterectomy, peritoneal cytology and pelvic lymphadenectomy. In the case of abdominal metastases, paraaortic lymphadenectomy, omentectomy and metastasectomy are indicated. This extended surgery is always indicated in patients with operable ovarian cancer [23, 24]. Nowadays, total laparoscopic hysterectomy (TLH) with lymphadenectomy is often carried out instead of laparotomy in the case of endometrial cancer. The benefits associated with this procedure are shorter hospital stay and fewer complications, but its main drawbacks are longer duration of surgery and more expensive equipment [25].

Guidelines for adjuvant treatment in patients with the synchronous cancers have not been yet established. In clinical practice, usually it is a compilation of guidelines for treatment of respective cancers. Chemotherapy is indicated in all patients with ovarian cancer with the exception of stage IA/B grade 1 . In patients with endometrial cancer, chemotherapy is indicated only when risk of distant metastases is high (grade 3, clear cell or papillary serous histological type - type II carcinomas, involvement of parametrium or metastases to lymph nodes). In patients with ovarian cancer, standard chemotherapy is based on platinum compound combined with paclitaxel, in patients with endometrial cancer, doxorubicin is also recommended [24]. An important part of adjuvant treatment in endometrial cancer is radiotherapy, which is indicated in patients with stage IA G3 or in stages IB-II [23, 24]. In patients at stages I-II with other negative prognostic factors, who are treated with adjuvant radiotherapy, sometimes chemotherapy is also considered. Adjuvant external beam radiotherapy is efficient against pelvic failures, unfortunately it is associated with severe early and late toxicity. Adjuvant vaginal brachytherapy is a good tool to prevent vaginal recurrences, but postoperative irradiation does not improve overall survival $[15,26,27]$.

The choice of adjuvant therapy in most cases of synchronous cancers depends on the stage and grade of ovarian cancer, because this neoplasm is characterized by worse prognosis and greater risk of recurrence. In patients with stage IA uterine cancer, a 5-year risk of recurrence is lower than $10 \%$, in patients with higher grading (G2-3) or in stage IB-IIIA it is estimated at 15\% [28]. Historical data show that in patients with ovarian cancer, who underwent operation without chemotherapy, a 5 -year survival rate was $67 \%$ in stage I, $24 \%$ in stage II and $1 \%$ in stages III and IV [29].

The use of radiotherapy in patients with the synchronous cancers, who are treated with adjuvant chemotherapy, is controversial. According to Maggi et al. [30], patients with endometrial cancer and a high risk of relapse (stages IB-II, grade G3 or stage III), gained the same benefit regarding overall survival and recurrence-free survival from chemotherapy with cisplatin, doxorubicin and cyclophosphamide as from radiotherapy. Radiotherapy delayed local relapses and chemotherapy delayed metastases but differences were not statistically significant. However, Susumu et al. [31] reported that adjuvant chemotherapy was more beneficial than radiotherapy in high-risk patients.

On the other hand, Hogberg et al. [32] on the basis of 2 clinical trials NSGOEC-9501/EORTC-55991 and MaNGO ILIADE-III comparing sequential adjuvant chemoradiotherapy with radiotherapy in patients with operable endometrial cancer and a high risk of recurrence showed that combined treatment prolongs recurrencefree survival and cancer-specific survival but these trials included patients with single endometrial cancer and chemotherapy was different than paclitaxel-carboplatin combination.

According to different authors, $60-80 \%$ of patients with synchronous cancers were treated with adjuvant chemotherapy, 2-13\% - with radiotherapy, 4-28\% with chemotherapy and radiotherapy and $2 \%$ - were without adjuvant treatment $[6,16]$.

All patients in our series received adjuvant chemotherapy because of ovarian cancer, none of them had adjuvant radiotherapy. 
According to different authors, a 5-year survival rate in patients with the synchronous cancers is $71-96 \%$ [6, 16-20]. In Oranratanaphan's et al. [21] study, a 5-year recurrence-free survival rate and overall survival rate of patients with the synchronous cancers and patients with single endometrial cancer were $64.2 \%$ vs. $41.5 \%$, $(p=0.17)$ and $92.8 \%$ vs. $48.5 \%(p=0.036)$, respectively.

In our series, median follow up was 13 months (range: 3-53 months). One patient experienced relapse, one was lost to follow up. Other patients are alive without relapse. For comparison, in patients with ovarian cancer from our district a 5-year disease-free survival rate and overall survival rate are $61.3 \%$ and $48.1 \%$, respectively [22].

\section{Conclusions}

Synchronous ovarian and uterine cancers are predominant among patients with multiple gynecological cancers. The synchronous cancers are usually diagnosed at an earlier stage, have lower grading and prognosis is better when compared to a single advanced cancer. Diagnosis at an early stage is most likely possible due to symptoms caused by uterine cancer. The most frequent histological type in both localizations is endometrioid cancer. This article presents 10 patients with synchronous ovarian and endometrial cancers, whose courses of the disease were concordant with data from the literature. The differentiation of synchronous cancers from a single disseminated neoplasm is important, because it enables optimal adjuvant treatment, which further improves prognosis.

\section{Disclosure}

Authors report no conflicts of interest.

\section{References}

1. Tong S, Lee Y, Park J, et al. Clinical analysis of synchronous primary neoplasms of the female reproductive tract. Eur J Obstet Gynecol Reprod Biol 2008; 136: 78-82.

2. Eisner R, Nieberg R, Berek J. Synchronous primary neoplasms of the female reproductive tract. Gynecol Oncol 1989; 33: 335-339.

3. Gungor T, Kanat-Pektas M, Ustunyurt E, et al. Synchronous primary tumors of the female genital tract: a single center experience. Arch Gynecol Obstet 2009; 279: 667-672.

4. Huang Y, Hung Y, Yeh L, et al. Synchronous ovarian endometrioid adenocarcinoma and endocervical mucinous adenocarcinoma. Taiwan J Obstet Gynecol 2006; 45: 264-267.

5. Williams M, Bandera E, Demissie K, et al. Synchronous primary ovarian and endometrial cancers: a population-based assessment of survival. Obstet Gynecol 2009; 113: 783-789.

6. Lim YK, Padma R, Foo L, et al. Survival outcome of women with synchronous cancers of endometrium and ovary: a 10 year retrospective cohort study. J Gynecol Oncol 2011; 22: 239-243.

7. Matias-Guiu X, Bussaglia E, Catasus L, et al. Correspondence re: W.M. Lin et al., loss of heterozygosity and mutational analysis of the PTEN/MMAC1 gene in synchronous endometrial and ovarian carcinomas. Clin. Cancer Res., 4: 2577-2583, 1998. Clin Cancer Res 2000; 6: 1598-1600.

8. Lin WM, Forgacs E, Warshal DP, et al. Loss of heterozygosity and mutational analysis of the PTEN/MMAC1 gene in synchronous endometrial and ovarian carcinomas. Clin Cancer Res 1998; 4: 2577-2583.
9. Badowska-Kozakiewicz AM. Wybrane markery nowotworowe w rutynowej diagnostyce raka endometrium i szyjki macicy. Prz Menopauz 2012; 16: 168-173.

10. Zając A, Stachowiak G, Stetkiewicz T, et al. Gen TP53 i białko p53 a rak błony śluzowej trzonu macicy. Prz Menopauz 2013; 17: 228-230.

11. Furlan D, Carnevali I, Marcomini B, et al. The high frequency of de novo promoter methylation in synchronous primary endometrial and ovarian carcinomas. Clin Cancer Res 2006; 12: 3329-3336.

12. Serkies K, Sinacki M, Jassem J. The role of hormonal factors and endocrine therapy in ovarian cancer. Contemp Onkol (Pozn) 2013; 17: 14-19.

13. Soliman PT, Broaddus RR, Schmeler KM, et al. Women with synchronous primary cancers of the endometrium and ovary: do they have Lynch syndrome? J Clin Oncol 2005; 23: 9344-9350.

14. Zając A, Stachowiak G, Pertyński T. Association between MDM2 SNP309 polymorphism and endometrial cancer risk in Polish women. Pol J Pathol 2012; 63: 278-283.

15. Kellas-Ślęczka S, Wojcieszek P, Białas B. Adjuvant vaginal brachytherapy as a part of management in early endometrial cancer. J Contemp Brachytherapy 2012; 4: 247-252.

16. Ma SK, Zhang HT, Sun YC, et al. Synchronous primary cancers of the endometrium and ovary: review of 43 cases. Zhonghua Zhong Liu Za Zhi 2008; 30: 690-694.

17. Caldarella A, Crocetti E, Taddei GL, et al. Coexisting endometrial and ovarian carcinomas: a retrospective clinicopathological study. Pathol Res Pract 2008; 204: 643-648.

18. Signorelli M, Fruscio R, Lissoni AA, et al. Synchronous early-stage endometrial and ovarian cancer. Int J Gynaecol Obstet 2008; 102: 34-38.

19. Natee J, Kietpeerakool C, Srisomboon J, et al. Clinicopathologic analysis of women with synchronous primary carcinomas of the endometrium and ovary: 10-year experience from Chiang Mai University Hospital. Asian Pac J Cancer Prev 2006; 7: 234-238.

20. Zaino R, Whitney C, Brady MF, et al. Simultaneously detected endometrial and ovarian carcinomas - a prospective clinicopathologic study of 74 cases: a gynecologic oncology group study. Gynecol Oncol 2001; 83: 355-362.

21. Oranratanaphan S, Manchana T, Sirisabya N. Clinicopathologic variables and survival comparison of patients with synchronous endometrial and ovarian cancers versus primary endometrial cancer with ovarian metastasis. Asian Pac J Cancer Prev 2008; 9: 403-407.

22. Czernek U. Rak jajnika - ocena skuteczności leczenia chorych z makroregionu tódzkiego w zależności od wybranych cech klinicznych i patologicznych. Praca na stopień doktora nauk medycznych, Łódź 2008.

23. Colombo N, Preti E, Landoni F, et al. Endometrial cancer: ESMO Clinical Practice Guidelines for diagnosis, treatment and follow-up. Ann Oncol 2011; 22: vi35-39.

24. Zalecenia postępowania diagnostyczno-terapeutycznego w nowotworach złośliwych - $2011 \mathrm{r}$. Tom 1. Krzakowski M, Jassem J (eds.). $1^{\text {st }}$ ed. Via Medica, Gdańsk 2011.

25. Majchrzak-Baczmańska D, Antosiak B, Malinowski A. Operacyjne leczenie raka endometrium: laparotomia czy laparoskopia? Prz Menopauz 2013; 17: 125-131.

26. Kuo HC, Mehta KJ, Yaparpalvi R, et al. Feasibility study and optimum loading pattern of a multi-ring inflatable intravaginal applicator. J Contemp Brachytherapy 2013; 5: 93-100.

27. Donnelly ED, Rakhra S, Helenowski I, et al. Dosimetry and toxicity outcomes in postoperative high-dose-rate intracavitary brachytherapy for endometrial carcinoma. J Contemp Brachytherapy 2012; 4: 135-140.

28. The Chemotherapy Source Book. Perry MC (ed.). $4^{\text {th }}$ ed. Lippincott, Philadelphia 2008.

29. Young RC, Fisher RI. The staging and treatment of epithelial ovarian cancer. Can Med Assoc J 1978; 119: 249-256.

30. Maggi R, Lissoni A, Spina F, et al. Adjuvant chemotherapy vs radiotherapy in high-risk endometrial carcinoma: results of a randomised trial. $\mathrm{Br}$ J Cancer 2006; 95: 266-271.

31. Susumu N, Sagae S, Udagawa Y, et al. Randomized phase III trial of pelvic radiotherapy versus cisplatin-based combined chemotherapy in patients with intermediate- and high-risk endometrial cancer: a Japanese Gynecologic Oncology Group study. Gynecol Oncol 2008; 108: 226-233.

32. Hogberg T, Signorelli M, de Oliveira CF, et al. Sequential adjuvant chemotherapy and radiotherapy in endometrial cancer - results from two randomised studies. Eur J Cancer 2010; 46: 2422-2431. 